\title{
Endovascular Treatment of Thoracic Dissection
}

\author{
H. Rousseau, M.D., ${ }^{1}$ O. Cosin, M.D., ${ }^{1}$ B. Marcheix, M.D., ${ }^{1}$ V. Chabbert, M.D., ${ }^{1}$ \\ M. Midulla, M.D., ${ }^{1}$ C. Dambrin, M.D., ${ }^{1}$ C. Cron, M.D. ${ }^{1}$ B. Leobon, M.D., ${ }^{1}$ \\ C. Conil, M.D., ${ }^{1}$ P. Massabuau, M.D.,' ${ }^{1}$ P. Otal, M.D., ${ }^{1}$ and F. Joffre, M.D.'
}

Type A aortic dissection remains fatal if untreated. Although classical medical therapy for type B dissection is considered the therapy of choice in uncomplicated cases, the paradigm is changing as greater experience is accrued with endovascular treatments and technical advances improve the long-term outlook. Diagnosis is also becoming more sophisticated, allowing greater appreciation of the anatomy of dissections and improving the knowledge base as their natural history is assessed.

KEYWORDS: Aortic dissection, stent graft, multidetector computed tomography, transesophageal echocardiography

\begin{abstract}
Objectives: Upon completion of this article, the reader should understand the diagnostic and therapeutic approaches to acute aortic syndromes, in particular the new endovascular techniques for the treatment of descending thoracic aortic dissections.

Accreditation: Tufts University School of Medicine (TUSM) is accredited by the Accreditation Council for Continuing Medical Education to provide continuing medical education for physicians.

Credit: TUSM designates this educational activity for a maximum of 1 AMA PRA Category 1 Credit $^{\text {TM }}$. Physicians should only claim credit commensurate with the extent of their participation in the activity.
\end{abstract}

Since its first description $>250$ years ago, the associated morbidity and mortality of thoracic aortic dissections remain high despite improvements in medical and surgical treatment. The recently adopted term acute aortic syndrome includes aortic dissections, intramural hematomas (IMHs), and symptomatic aortic ulcers.

Recent advances in imaging and endovascular therapeutic techniques have further emphasized the importance of early diagnosis of this syndrome to improve survival rates. New imaging modalities, such as transesophageal echocardiography (TEE) and multidetector computer tomography (MDCT), allow a better and earlier diagnosis of aortic diseases in urgent situations. Valuable information about the location of entry tears and the extension of the dissection is crucial for therapeutic strategy and subsequent prognosis. The introduction of endovascular techniques, especially aortic endografts, has led to reconsideration of classic therapeutic options.

In this chapter, we review the diagnostic and therapeutic approaches to acute aortic syndromes, in particular the new endovascular techniques for the treatment of descending thoracic aortic dissections.
${ }^{1}$ Department of Radiology, Cardio-Vascular Surgery and Cardiology, Hôpital Rangueil, Toulouse, France.

Address for correspondence and reprint requests: H. Rousseau, M.D., Department of Radiology, Hôpital Rangueil, 1, avenue Jean-Poulhes, TSA 50 032, 31059, Toulouse cedex 9, France.

Aorta and Great Vessels; Guest Editor, Tony Nicholson, B.Sc.,
M.Sc., M.B., Ch.B., F.R.C.R.

Semin Intervent Radiol 2007;24:167-179. Copyright (C) 2007 by Thieme Medical Publishers, Inc., 333 Seventh Avenue, New York, NY 10001, USA. Tel: +1(212) 584-4662.

DOI 10.1055/s-2007-980041. ISSN 0739-9529. 


\section{PHYSIOPATHOLOGY}

The term acute aortic syndrome includes three entities with similar symptoms and therapeutic approaches: the classic aortic dissection, the intramural hematoma (IMH), and the symptomatic aortic ulcer.

Aortic dissection is typically characterized by a longitudinal cleave in the media. A cleavage plane or false lumen is usually located between the middle and external layer of the media and communicates with the aorta through one or several intima-medial tears (reentries). Propagation of the dissection can proceed in an anterograde or retrograde fashion from the initial tear. It may involve side branches and cause complications such as malperfusion syndromes, pericardial tamponade, or aortic valvular insufficiency. ${ }^{1}$ In the general population, studies suggest an incidence of aortic dissection of 2.6 to 3.5 cases per 100,000 person-years. ${ }^{1-3}$ A review of 464 patients from the International Registry of Acute Aortic Dissection (IRAD) showed that two thirds were men, with a mean age of 63 years. ${ }^{4}$ Women appeared less frequently affected by acute aortic dissection, and if so, at older ages (mean age, 67 years). ${ }^{5}$ Type $\mathrm{A}$ dissections are twice as common as type B.

Aortic IMH is considered a precursor of dissection. It originates from ruptured vasa vasorum in medial wall layers, resulting in an aortic wall infarct that may provoke a secondary tear, and subsequently a classic aortic dissection. ${ }^{6}$ As with dissections, IMH also may extend, progress, regress, or resorb. The most frequent location is the descending aorta (50 to $85 \%)$. IMH is typically associated with hypertension. ${ }^{7}$

Penetrating atherosclerotic ulcer (PAU) can lead to IMH, aortic dissection, or perforation. ${ }^{8,9}$

Both acquired and genetic conditions share a common pathway leading to the breakdown in the integrity of the intima. All mechanisms that weaken the media layers of the aorta eventually lead to higher wall stress, which can induce aortic dilation and aneurysm formation, resulting in intramural hemorrhage, aortic dissection, or rupture. The factors culminating in a clinical dissection are quite diverse. The most common risk condition for aortic dissection is hypertension, with chronic exposure of the aorta to high pressures leading to intimal thickening, fibrosis, calcification, and extracellular fatty acid deposition. Marfan's syndrome, vascular Ehlers-Danlos syndrome, annuloaortic ectasia, bicuspid aortic valve, and familial aortic dissection are genetic conditions that often cause acute aortic syndromes. A common denominator to these different genetic disorders is a similar pathophysiology that includes a dedifferentiation of vascular smooth muscle cells and enhanced elastolysis of aortic wall components, leading to a compromised intima and aortic dissection. $^{10}$

Two classifications are currently used based on anatomic criteria. According to DeBakey's classification, type I dissections begin in the proximal aorta and involve both the ascending and descending thoracic aorta, type II is confined to the ascending aorta, and type III is confined to the descending aorta. ${ }^{11}$ The Stanford classification, which is more frequently used, describes a type A dissection with the tear in the ascending aorta requiring urgent surgery, and a type B dissection in which the tear originates in the descending thoracic aorta ${ }^{12}$ The Stanford classification neither describes involvement of the aortic arch nor the extension of the dissection. Approximately 60 to $70 \%$ of aortic dissections are type $A$.

In a small subset of type $\mathrm{A}$ dissections, the entry tear is in the descending aorta and propagation occurs retrogradely up to the ascending aorta (retrograde type A). The incidence of this subtype ranges from 10 to $27 \%$ among DeBakey type III dissections and from 4 to $20 \%$ among Stanford type A dissections. ${ }^{12-14}$ Emergent surgical treatment is still indicated for this subtype, although this remains challenging and controversial.

Aortic dissections are also classified according to the time passed after presentation. Dissections present for $<2$ weeks are considered acute; those older than 2 weeks are described as chronic.

\section{Acute Dissections}

Acute type A dissection is highly lethal, with a mortality of 1 to $2 \%$ per hour early after symptom onset. ${ }^{15}$ The risk of death for type A dissection is increased in patients who present with or develop complications such as pericardial tamponade, involvement of coronary arteries causing acute myocardial ischemia/infarction, or malperfusion of the brain. ${ }^{1}$ Other predictors of increased inhospital death include age $\geq 70$ years, hypotension, cardiac tamponade, kidney failure, and pulse deficits. ${ }^{16}$ Data from the IRAD registry of acute aortic dissection (645 patients stratified by medical and surgical treatment in both type $\mathrm{A}$ and $\mathrm{B}$ aortic dissection) showed that without immediate surgical repair, medical management alone is associated with a mortality of nearly $24 \%$ by 24 hours after presentation, $29 \%$ by 48 hours, $44 \%$ by day 7 , and $49 \%$ by 14 days. ${ }^{4}$ Even with surgical repair, inhospital mortality rates are $10 \%$ by 24 hours, $12 \%$ by 48 hours, $16 \%$ by 7 days, and nearly $20 \%$ by 14 days. ${ }^{16}$ At 2 weeks, mortality curves of untreated aortic dissections begin to plateau.

Patients with acute type $\mathrm{B}$ dissection tend to be older and present a higher surgical risk, but conversely they are at lower risk of early death from complications. These complications determine the clinical outcome: rupture, ischemic events, and aneurysmal enlargement of the false channel. The mortality rate of aortic rupture is $>60 \%$ in type $\mathrm{B}$ dissection. ${ }^{17} \mathrm{~A}$ large retrospective series of uncomplicated type B dissection patients from Duke and Stanford suggested equivalent outcome with 
medical and surgical treatment. ${ }^{18}$ The IRAD study revealed in-house mortality rates of $11 \%$ and $31 \%$ for medical and surgical treatment groups, respectively. ${ }^{4}$ Surgical treatment is also associated with significant morbidity, particularly paraplegia (7 to $36 \%) .{ }^{19,20} \mathrm{~Pa}^{-}$ tients with uncomplicated type $\mathrm{B}$ dissection have a 30day mortality of $10 \%$, but patients who develop ischemic complications or contained rupture often require urgent aortic repair, which carries a mortality of $20 \%$ by day 2 and $25 \%$ by day $30 .{ }^{4}$

Consequently, the preferred treatment for most patients has been medical in the form of aggressive antihypertensive treatment. Surgical intervention is generally reserved for those who develop complications such as rupture, dissection progression, aneurysmal growth, refractory hypertension, intractable pain, and malperfusion syndromes from aortic branch vessel obstruction. ${ }^{21}$ Similar to type A dissection, advanced age, rupture, shock, and malperfusion are important independent predictors of early mortality. ${ }^{5,22}$

Ischemic complications are encountered in 30 to $50 \%$ of patients and determine one of the major indications for surgical intervention. ${ }^{23}$ The most commonly affected arteries are the iliac vessels. However, the most serious complications are associated with renal and/or mesenteric ischemia. Obstruction of aortic side branches can be caused by two different mechanisms, which can occur simultaneously as well (Fig. 1).
Static vascular obstruction is the extension of the dissection to the level of the affected side branch. The false lumen can thrombose at the level of the side branch and compress the true lumen, thus inducing outflow obstruction. In other cases the aortic side branches are perfused through a reentry, thereby reperfusing the true lumen because of subsequent outflow. Dynamic obstruction occurs secondary to compression of one of the two aortic channels, usually the true lumen without extension of the intimal flap in the affected side branches. Compression of the true lumen can be accompanied by a sort of prolapse of the intimal layer into the ostium of the nondissected side branch. The two mechanisms can be associated. The mortality rate of patients with renal ischemia is 50 to $70 \%$ and as high as $87 \%$ in mesenteric ischemia. ${ }^{24-26}$ Although the surgical success rate at reversing peripheral pulse deficits is high, the surgical in-hospital mortality rates in the setting of end-organ ischemia remain as high as $50 \%$ and $89 \%$ for renal and mesenteric ischemia, respectively. ${ }^{14,27,28}$

\section{Chronic Dissections}

With chronicity, the most common complication is the development of aneurysmal dilation of the false lumen occurring in 14 to $20 \%$ of patients. ${ }^{24}$ However, note that because medical therapy alone does not stop blood flow to the false lumen, 20 to $50 \%$ of patients who survive the

A
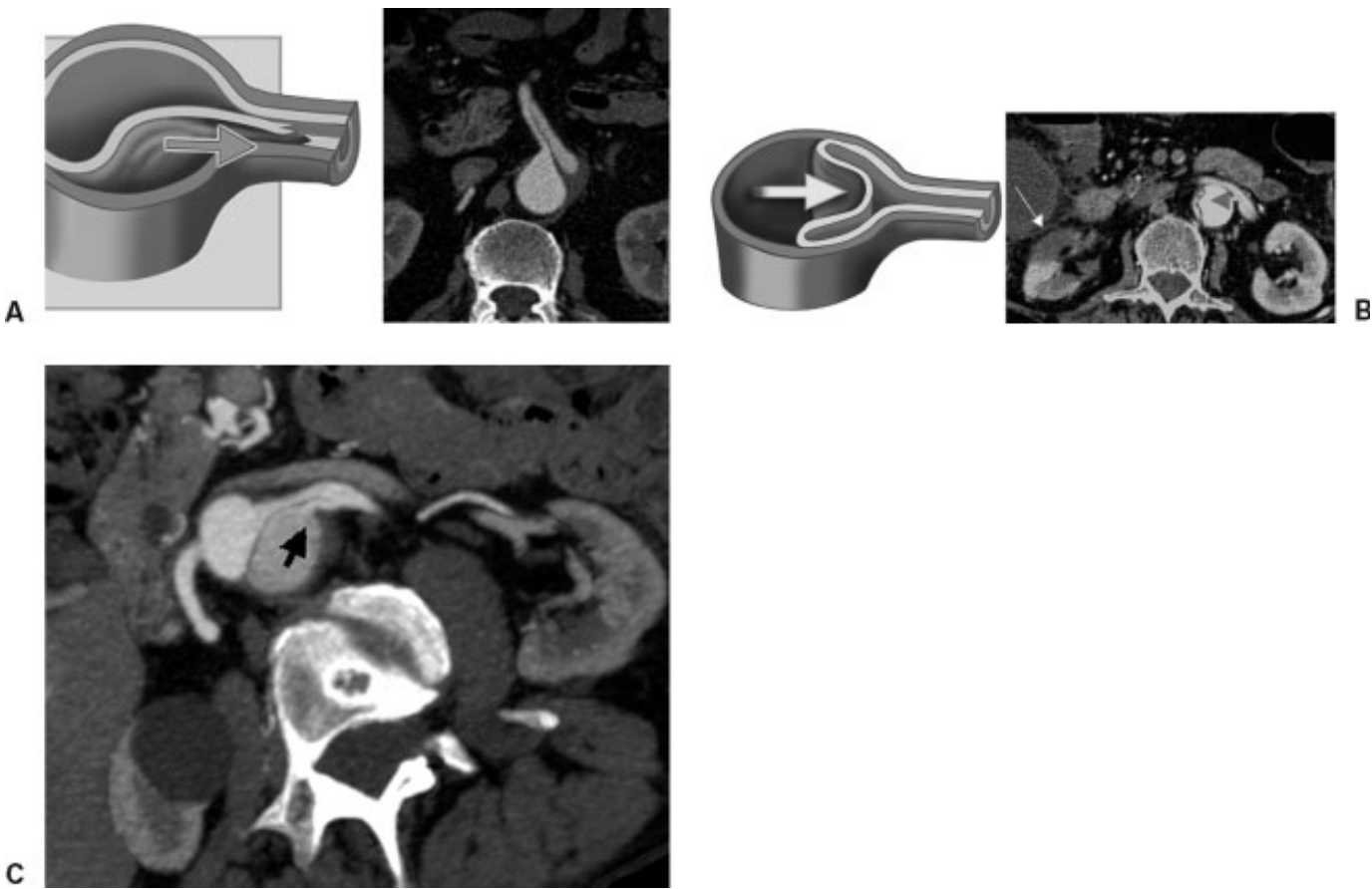

Figure 1 End-organ and lower limb ischemia: mechanisms of visceral and iliac arterial obstruction. (A) Static obstruction: Dissection flap extends into the superior mesenteric artery. If a reentry tear is present, distal perfusion is maintained. (B) Dynamic obstruction: True lumen has been compressed by false lumen (arrowhead) and right renal malperfusion is observed (arrow). In case of dynamic mechanism, perfusion could come either from true or false lumen. (C) The two mechanisms can coexist. In this case the true lumen of the left renal artery is compressed by the false lumen of the aorta (dynamic obstruction), but the true lumen is also perfused by the false lumen through a reentry (arrow) (static dissection), with subsequent outflow. (The author thanks Dr. F. Thony for the drawings.) 
acute phase develop aneurysmal dilation of the false lumen within 1 to 5 years after onset. ${ }^{29-31}$ Studies of the long-term follow-up of type B aortic dissection demonstrate growth rates of thoracic and abdominal aorta of 4.1 and $1.2 \mathrm{~mm}$ per year, respectively. ${ }^{32}$ The presence of blood flow in the false lumen was the only significant risk factor for increase in the diameter in the univariate and multivariate analysis $(3.3 \mathrm{~mm} / \mathrm{y}$ in the group with blood flow in the false lumen versus $-1.4 \mathrm{~mm} / \mathrm{y}$ in the group without blood flow, $P<0.0001)^{32}$

\section{Intramural Hematoma}

The treatment paradigm for IMH parallels the approach to classical aortic dissection. A meta-analysis of $11 \mathrm{IMH}$ studies found cumulative mortality for type A IMH to be $24 \%$ for those treated surgically, $47 \%$ for those treated medically, and 34\% overall. Mortality for type B IMH was $14 \%$ overall with little difference between surgical (15\%) and medical (13\%) treatment groups. ${ }^{33}$ Until further studies are definitive, many experts recommend aortic repair for acute IMH of the ascending aorta similar to type A dissection and aggressive medical therapy for IMH in the descending aorta similar to type $\mathrm{B}$ dissection. ${ }^{34}$

\section{Penetrating Atherosclerotic Ulcer}

At present, no consensus therapeutic strategy exists, although a more aggressive surgical approach, independent of location, is being increasingly considered especially in symptomatic patients. ${ }^{35,36}$

\section{TREATMENT}

The initial treatment of acute aortic syndrome aims to lower arterial blood pressure (systolic pressure, $110 \mathrm{~mm}$ $\mathrm{Hg})$ and heart rate $(60 / \mathrm{min})$. Further treatment depends on where the dissection originates. There is a general consensus in favor of surgical treatment for ascending aortic dissections. However, treatment of descending aortic dissections is still debated. ${ }^{12,18}$ As a whole, patients with uncomplicated aortic type $\mathrm{B}$ dissections are treated with medical therapy but may be considered candidates for endovascular therapy in the near future. Therefore, indications for operation in patients with acute type B aortic dissections are generally limited to the relief of life-threatening complications. ${ }^{14}$

Recently new endovascular methods, including implantation of aortic stent grafts (S-grafts), fenestration or implantation of noncovered stents, obviously less invasive than surgical techniques, have gone through an explosive technological development. ${ }^{37-40}$ The different endovascular techniques are complementary, the choice depending on the type of dissection, the clinical status of the patient, and the need to treat associated complications. The decision can only be taken after exact assessment of the whole affected aorta by means of MDCT, TEE, or magnetic resonance angiography.

\section{STENT GRAFTS}

The concept of endovascular stent-graft repair of aortic dissection is based on successful placement of the device over the primary entry tear to obliterate blood flow into the false lumen and to redirect the flow into the true lumen. As demonstrated in experimental models of dissection, coverage of the primary entry tear is the optimal method of relieving true lumen collapse and concomitantly promotes thrombosis of the false lumen, distally, independent of the location of the primary intimal disruption. ${ }^{41}$ The tempo of false lumen thrombosis is variable and influenced by several factors, such as the size of the false lumen, the length of the stent graft, and the amount of residual false lumen flow via uncovered additional tears or collaterals, in particular from the left subclavian artery. From the literature, we know that dissections with naturally thrombosed false lumen are associated with improved prognosis. ${ }^{13,42}$ False lumen patency, in contrast, contributes to progressive aortic dilation and is a predictor of late mortality. ${ }^{32,43}$ Protection against thoracic false lumen aneurysm formation might be expected to be positive. Complete exclusion of the thoracic false lumen with a remodeling of the aorta is the ultimate goal. As this happens, by redirecting the flow in the true lumen, downstream branch vessel ischemia is resolved, in particular in acute dynamic obstruction where reversal of ischemia occurs expeditiously after stent graft placement. $^{38}$

Different devices are available in Europe. All stent grafts are self-expanding and constrained in a sleeve or sheath. They are made of a metallic Nitinol skeleton (except for the stainless-steel Cook device), covered by a membrane of either polytetrafluoroethylene (Gore and Endofit devices) or polyester (Medtronic, Jotec, Bolton, and Cook devices). Proximal or distal ends of the graft can be fully covered or not. Medtronic, Endofit, Bolton, and Jotec devices use bared stent ends to better anchor to the aortic wall. The Gore and Cook devices are fully covered, but Cook's possesses metallic barbs for better fixation. Finally, new grafts with fenestrations or branches are being evaluated.

The deployment of stent grafts varies depending on the device used In general, while holding the stent graft stationary, they are deployed either by withdrawing the delivery sheath (Medtronic, Cook, Endofit, Bolton, and Jotec) or by pulling a string that releases the stent graft sleeve (Gore). Commercially available thoracic stent grafts do not significantly shorten during or after deployment. 


\section{Preinterventional Planning}

Pretreatment imaging is crucial for evaluating patient suitability for endovascular treatment, selecting the appropriate stent graft, and planning the intervention. MDCT with 3D reconstructions is probably the best anatomical evaluation method for the aorta as well as the iliac and femoral arteries where diameter, degree of calcification, and tortuosity are very important. Magnetic resonance (MR) imaging is also a good alternative especially in patients with renal failure, however, due to the lower spatial definition of MR, its inability to image calcium, its less open access, and its frequent incompatibility with life support and monitoring equipment, MDCT is preferred. Angiography using a calibrated catheter is less useful than MDCT but could help estimate the lesion length and aortic angulations in some complex cases.

Stent graft diameter should be oversized by 10 to $15 \%$ (based on MDCT or MR measures of the diameter of a nondissected part of the aorta, at the arch in most of the cases) to provide good apposition to the aortic wall. At least $1.5 \mathrm{~cm}$ of normal aorta is required to provide a good seal, and the length of the device should be long enough to cover a substantial part of the dissected aorta. Because the aim is to exclude the entry site and redirect the flow to the true lumen, it is not necessary to cover the entire affected aorta.

\section{Stent Graft Placement}

Endovascular stent placement procedures are performed via the femoral or external iliac approach under general anesthesia. When the iliac arteries are too tortuous or too small, vascular access may be achieved through the common iliac artery or the infrarenal aorta using a temporary graft to facilitate vascular access.

Ideally, the vascular procedure must be done in an angio suite equipped and kept aseptic as an operating room. Cardiopulmonary bypass should be available during the examination. The procedure should be monitored under digital subtraction angiography. TEE, although not mandatory, may sometimes be useful because it allows assessment of the true and false aortic lumen and especially the origin of the dissection. The latter might be difficult to visualize with angiography. In addition, TEE also provides information about exclusion of the false lumen, the diameter of both the false and true lumen, and the behavior of the aortic valve as well as left ventricular function.

A radiopaque marker (usually a $5 \mathrm{~F} 30-\mathrm{cm}$-long sheath) may be inserted via the left brachial artery to single out the ostium of the left subclavian artery. This marker at the ostium of the left subclavian artery (LSA) may be very useful for optimal stent placement in case of an isthmic lesion (Fig. 2). Coil embolization of the origin of the subclavian artery can be done from here if residual back flow is observed in the false lumen after stent graft deployment.

After administration of a 5000-IU bolus of heparin, the delivery system is inserted over a $260-\mathrm{cm}$-long guidewire (Back-Up Meier; Boston Scientific, Natick, MA; Amplatz; Boston Scientific, or Lunderquist; Cook, Bloomington, IN) through the transverse arteriotomy of the common femoral artery and advanced under fluoroscopy. Just before the device is released, vasodilators such as sodium nitroprusside can be administered intravenously to decrease the systolic pressure to $\sim 70 \mathrm{~mm} \mathrm{Hg}$. Adenosine-induced cardiac standstill has been proposed to ensure precise stent graft positioning but is not necessary. After correct positioning, the device is deployed. Except in chronic dissections, balloon inflation to mold the stent graft and obtain complete expansion should be avoided because some cases of aortic rupture have been described.

Completion angiography and, if used, TEE are performed, catheters removed and the arteriotomy repaired.

In our practice MDCT is performed at the time of discharge and thereafter at 3, 6, and 12 months and then annually. Chest radiographs are obtained at the same time intervals. For young patients or patients with renal failure, especially if they are diabetic, MRI followup should be recommended.

\section{TECHNICAL CONSIDERATIONS}

Short Neck When the neck is too short, a device with a proximal segment consisting of a bare stent can be placed across the LSA to effectively maximize the length of graft contact with the aortic wall proximal to the tear. Intentional exclusion of the LSA by the stent graft can be done without surgical transposition. If symptoms develop later (steal syndrome or left arm deficit), surgical revascularization can be performed electively in a second procedure. Many authors have reported no major complication after intentional LSA occlusion. However, before occluding the LSA, contralateral vertebral artery perfusion and collateral pathways should be assessed to avoid major neurological complication. ${ }^{44,45}$ LIMA coronary bypass, contralateral stenosis, or the absence of collaterals must be considered as a contraindication to intentional exclusion of the LSA without previous bypass.

Aneurysm Involves the Aortic Arch When the aneurysm involves the aortic arch, in particular for retrograde type B dissections, proximal placement of the stent graft requires some form of bypass to the left carotid artery, usually from a branch of the brachiocephalic artery. When the arch is completely involved, one needs to look even further for a source of inflow to the aortic branch vessels before deployment of the graft. 
A
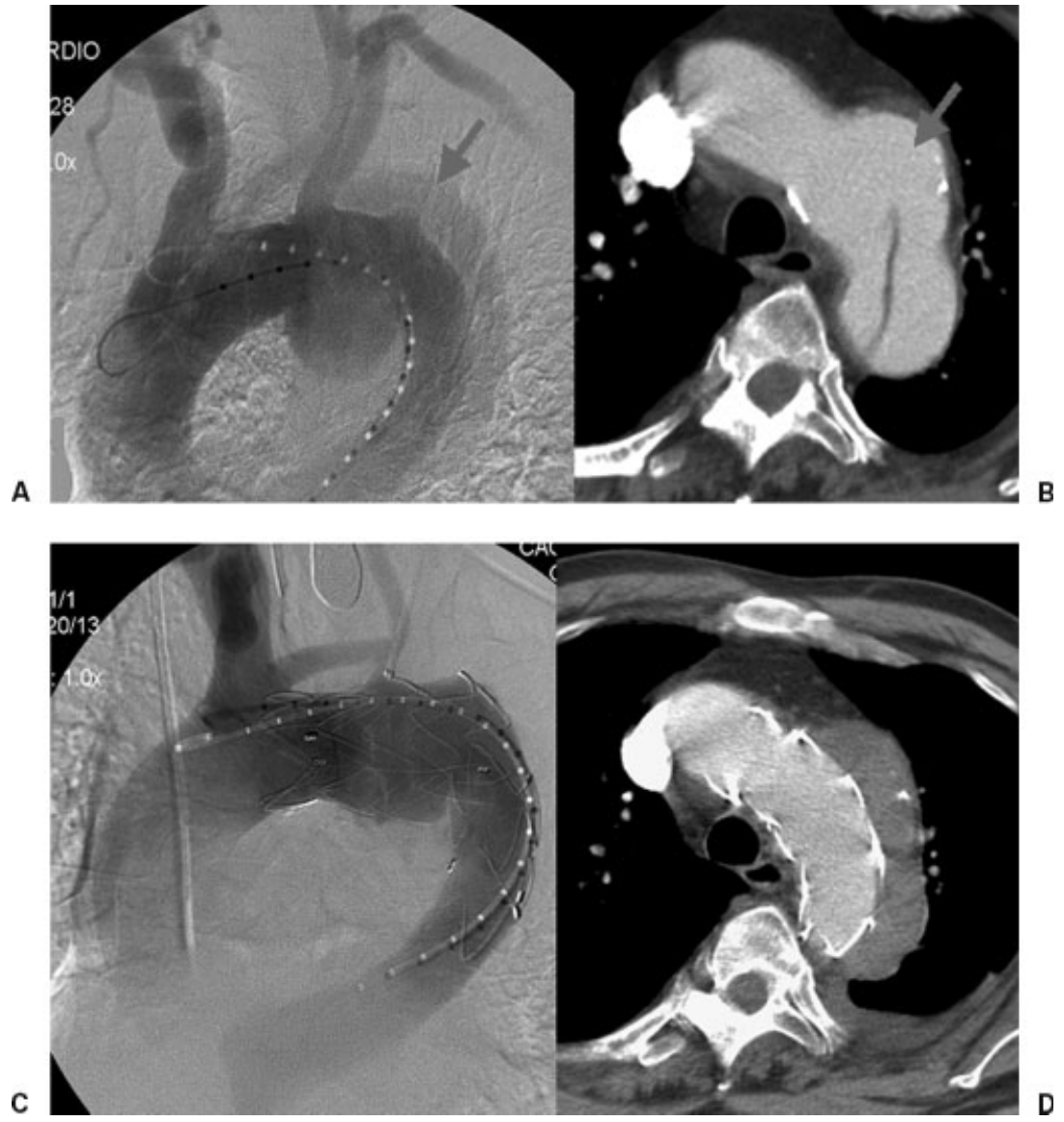

Figure 2 Typical acute type B dissection. (A) Angiography and (B) computed tomography clearly show the entry tear (arrow), just beyond left subclavian artery (LSA) origin with contrast filling of false lumen. (C,D) After S-graft deployment at the level of the tear, complete exclusion of the false lumen is achieved and normal flow is reestablished into the true lumen. LSA origin was intentionally covered to gain a better proximal sealing. We note the lack of flexibility in the first generation's stent grafts while we are expecting a better remodeling performance with more flexible devices of the next generation.

Endovascular treatment in this situation is an appealing alternative but must deal with several challenging anatomical difficulties. The aortic arch is large, mobile, curved, and subject to high flow, and its main branches show little tolerance to ischemia.

\section{FENESTRATION OF DISSECTION FLAPS}

Percutaneous balloon fenestration is used to mitigate dynamic obstructions by creating an artificial tear in the flap to allow communication between the true and false lumens. ${ }^{46-48}$ Single or multiple fenestrations provide an improved balance with regard to hemodynamic forces within the true and false lumen and subsequently improve distal vascularization. An intravascular ultrasound (IVUS) catheter is placed in the largest lumen, usually the false lumen, to control the puncture of the intimal layer, which is performed with biopsy needles through the true lumen. The true lumen is usually entered by puncturing the side with the weaker or absent femoral pulse. After perforating the intimal wall, a guidewire is positioned and the fenestration is completed by means of inflating a 12 - to $14-\mathrm{mm}$ balloon or stenting at the puncture hole. ${ }^{37}$ For aortic dissections extending in the iliac arteries, a different fenestration technique could be used (the scissors technique). Two guidewires are introduced in both the false and true lumen of the aorta, and a single introducer is advanced over both wires, subsequently causing a longitudinal intimal tear and reestablishing distal outflow. This method has technical and financial benefits because it is executed without IVUS and balloon angioplasty or stenting. ${ }^{40}$ Fenestrations should be limited to the abdominal aorta. In the thoracic aorta, it only enlarges the entry hole and increases the pressure in the false lumen and compression of the true lumen, jeopardizing distal outflow. Because of embolic risks, fenestration is contraindicated if one of the channels is partially or totally thrombosed. In this case, noncovered stents can be used to enlarge the aortic true lumen.

\section{STENTS}

Stents are used to treat distal ischemic complications related to the static mechanism and reestablish blood flow distal to the origin of the dissection by decompressing the 
lumen. The stent shifts the dissection membrane toward the other lumen and mechanically improves distal perfusion. This technique requires detailed information about the role of the true and false lumen with respect to visceral and lower extremity vascularization. Deployment of an infrarenal aortic stent can be considered to treat lower limb ischemia, especially when the false lumen compressing the true lumen is thrombosed. Different noncovered stents, either self or balloon expandable, can be used for this application, but high radial force is necessary in most of the cases. ${ }^{49}$

\section{INDICATIONS AND RESULTS}

The aims of any treatment are to obtain thrombosis of the false lumen to prevent aortic rupture and to treat ischemic complications.

\section{Refixation of the Dissected Layers}

To prevent aortic rupture or progressive aneurysmal dilation in the acute dissection, a TSG in the true lumen, just at the level of the origin of the dissection (Fig. 3), ${ }^{50-52}$ can be considered. The endovascular techniques for aortic dissection are much more complicated compared with endovascular treatment of aneurysmal disease. Most often, the different channels are not circular, which complicates the choice of graft. In acute aortic dissection, the large discrepancy between the caliber of the true and false lumen might be the main obstacle to deploying the endograft in a relatively disease-free aorta. In these cases it is possible to implant the device proximal to the dissection, with coverage of the left subclavian artery.

With chronic dissection, endovascular treatment has been met with scepticism given the anatomical and hemodynamic complexity of such lesions. ${ }^{53}$ The thick

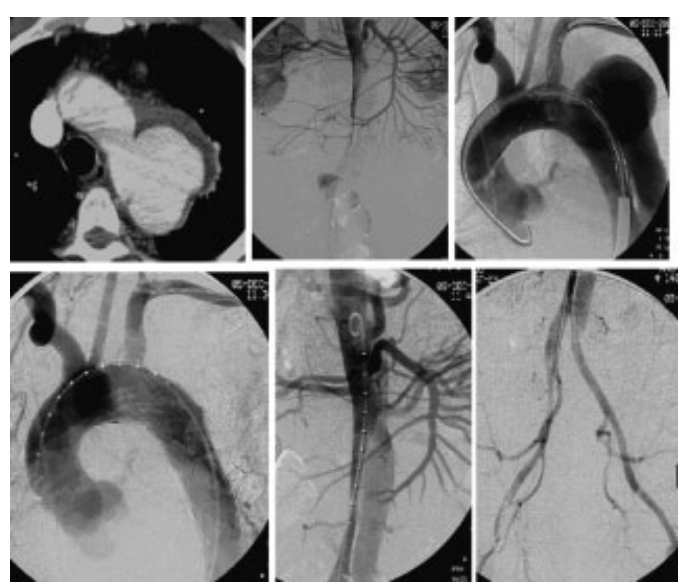

Figure 3 Patient with type B dissection developed acute lower limbs ischemia as a result of a compressed abdominal aortic true lumen by the false lumen. After a stent-graft covering of the proximal entry tear in the descending aorta, complete exclusion of the false lumen was observed. Concomitantly, normal perfusion of abdominal aortic lumen and iliac arteries was restored. and fibrotic nature of a chronic dissection flap may limit true lumen expansion following device placement. Additionally, multiple fenestrations between the true and false lumens often exist and may hinder false lumen thrombosis below the level of the TSG. Despite this, several groups have achieved good results with stentgraft treatment of chronic dissections. ${ }^{54-56}$ However, in a study by Kato and colleagues that evaluated both chronic and acute dissections, false lumen thrombosis was documented in all patients, but complete obliteration of the false lumen was found in only $38.5 \%$ of the chronic dissection patients versus $70 \%$ of the acute dissection patients (mean follow-up, 27 months). ${ }^{56}$

It is difficult to compare these preliminary results with the outcome of conventional surgery. The majority of surgical series show considerable morbidity and mortality, despite reimplantation of intercostal arteries, extracorporeal circulation, and profound hypothermia. In chronic type $\mathrm{B}$ dissections, mortality rate and neurological complications are $7.1 \%$ and $2.9 \%$, respectively, whereas in acute type B dissections these complications are $8.5 \%$ and $19 \%$, respectively. Age, cross-clamp time, and extent of involved intercostal arteries are the main risk factors for morbidity and mortality. ${ }^{57}$ Although the reports on TSG for type B dissection comprise a limited number of patients, the results are similar to those obtained for other indications. Nienaber et al described in a randomized study excellent results following endovascular treatment with exclusion of the false lumen at 3 months in all patients and no morbidity or mortality occurred..$^{39}$ A comparative group treated surgically had a mortality and morbidity rate of $33 \%$ and $42 \%$, respectively. Application of short endografts, not covering the distal descending thoracic aorta between T8-L2, might play an important role in the prevention of spinal cord ischemia. Other factors determining favorable neurological outcome are absence of aortic cross-clamping and duration of the procedure (TSG, $1.6 \pm 0.4$ hour; surgery, $8 \pm 2$ hours). ${ }^{39}$

A meta-analysis by Eggebrecht et al of 609 patients demonstrated a success rate of $>98 \%$ with $11.1 \%$ major complications, including $2.9 \%$ of neurological complications (1.9\% of cerebral vascular accident and $0.8 \%$ of paraplegia). ${ }^{58}$ The complication rate $(14.5 \%$ versus $8 \%$ ) and mortality at 30 days (9.8\% versus $3.2 \%)$ are significantly more important for the acute dissections than for chronic dissections. The death rate at 30 days is close to that observed after medical treatment according to IRAD, but it should be noted that the majority of the patients treated by TSG were treated for urgent or emergency complications where traditionally surgery is indicated, with a mortality of 50 to $88 \%$ in the presence of visceral ischemic complications and an average rate of paraplegia of $\sim 19 \%{ }^{4}$ After TSG in this situation, average surgical conversion rates are $1.1 \%$. Endovascular re interventions are necessary in $11.9 \%$ of cases on 
average, but recall that it is also the case in 11 to $20 \%$ of the patients medically treated and in $10.4 \%$ of the surgery patients. ${ }^{13,43,59}$ The actuarial curve of survival according to this meta-analysis is $90.6 \%$ to 6 months, $89.9 \%$ to 1 year, and $88.8 \%$ to 2 years. ${ }^{58}$

"Remodeling" of the aorta with retraction of the false channel and an expansion of the true channel is observed in $>75 \%$ of the cases if the false channel is completely excluded by TSG (Fig. 4).$^{60}$ It is well known from the literature that thrombosis of the false lumen is an essential factor in the outcome of medical treatment. ${ }^{32,43,61}$ For this reason, it appears essential to obtain total thrombosis of the false thoracic channel. This may require other endovascular interventions, such as embolization of the false lumen or collaterals (Fig. 5).

Whereas false lumen thrombosis is consistently observed at the level of the implanted stent graft, thrombosis distal to the device and particularly in abdominal false lumens is less common. It is thought that uncovered portions of a dissection flap oscillate with retrograde flow through distal flap disruptions, preventing false lumen thrombosis. This has implications regarding device length, and now, for these reasons, most investigators implant stent grafts that are clearly longer than before, usually in the range of 15 to $20 \mathrm{~cm}$ long. This added length confers an appearance to the aortic morphology after implantation that is more normal anatomically, especially in the arch, than that observed following placement of a short device focally over the entry tear. In addition, the longer device promotes more rapid tempo of thrombus formation within the proximal false lumen. However, extension of stent-graft coverage into the distal third of the descending thoracic aorta increases, theoretically, the risk of spinal cord ischemia. It has thus been suggested that distal extension with bare stents may provide a better structural stability to promote false lumen thrombosis without sacrificing intercostal flow. ${ }^{62}$

Significant controversy exists over the proper treatment for patients with acute retrograde type A dissection with an entry tear in the descending aorta. Kato and colleagues treated 10 retrograde type $\mathrm{A}$ patients without evidence of cardiac tamponade or severe aortic regurgitation using endovascular stent grafts. ${ }^{63,64}$ Entry closure and complete thrombosis of the false lumen of both the ascending and descending aorta was achieved in all patients. During mean follow-up of 20 months, all patients were alive and without rupture or aneurysm formation.

The placement of stent grafts in uncomplicated type $\mathrm{B}$ dissection is more controversial. Medical treatment was the only accepted treatment until stent grafts were used successfully, complicating the decision-making process. In light of the promising results, the INSTEAD trial (Investigation of STEnt grafts in patients with type B Aortic Dissection), a prospective,

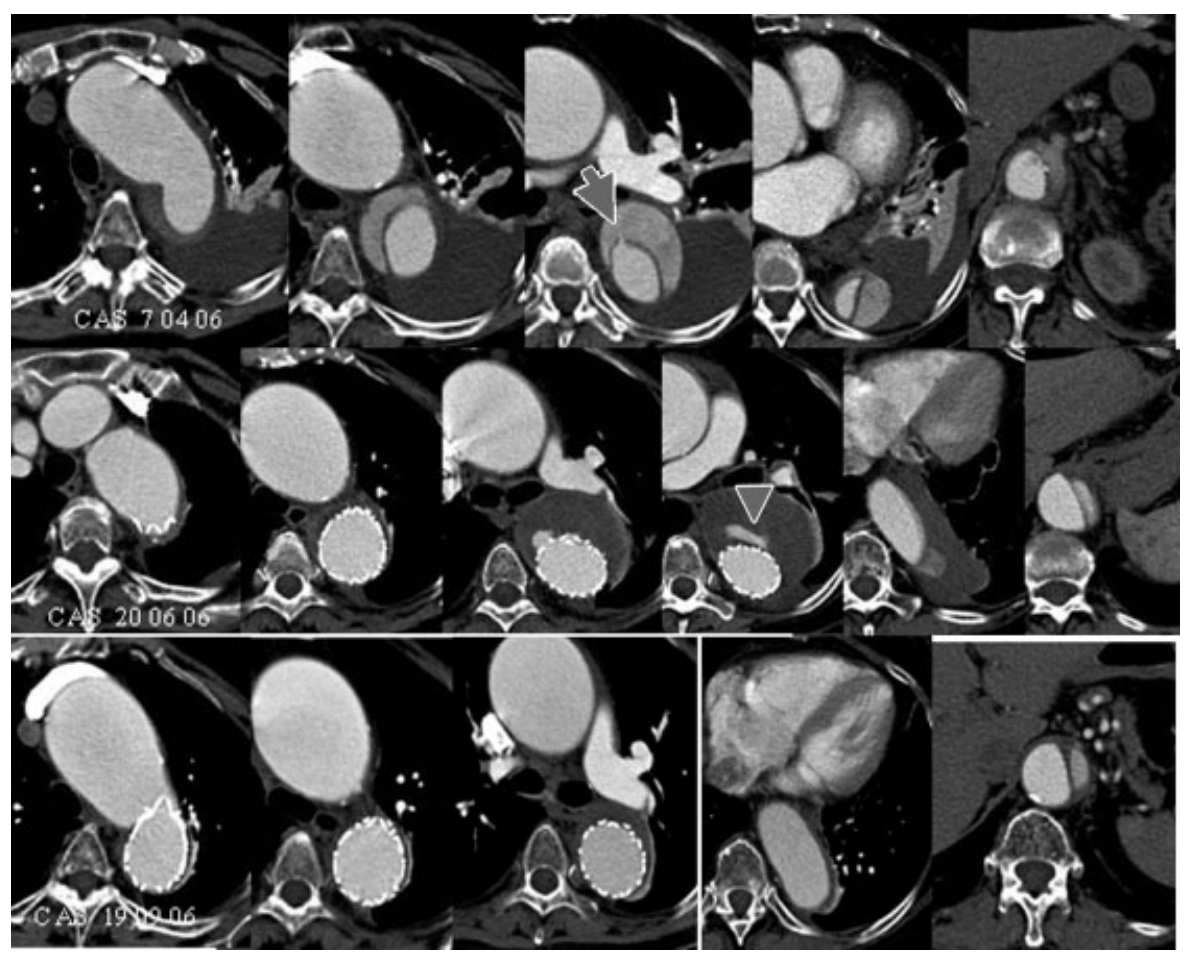

Figure 4 Type B dissection with entry tear (arrow) in the descending aorta treated by stent-graft insertion (preoperative, postoperative, and computed tomography scan at 3 months). Pathognomonic findings after successful exclusion of false lumen as a result of covering the entry tear: progressive expansion of the true lumen and parallel shrinking of the false lumen, defined as "aortic remodeling." A proximal small endoleak (arrowhead) persisted only at first follow-up and disappeared 3 months later. 
A
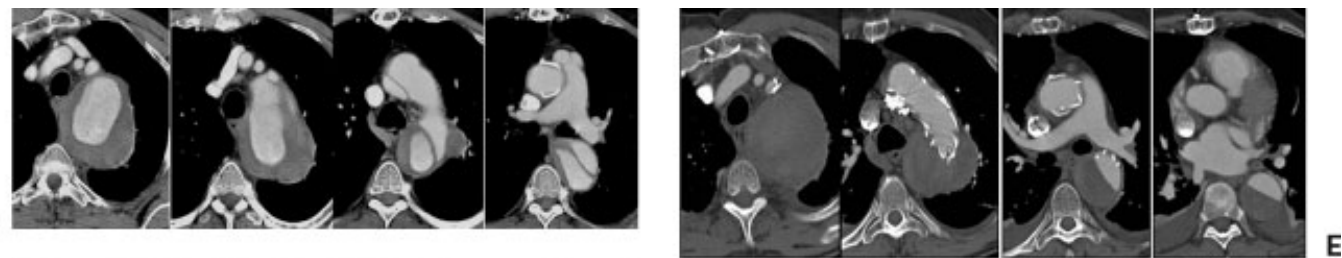

B

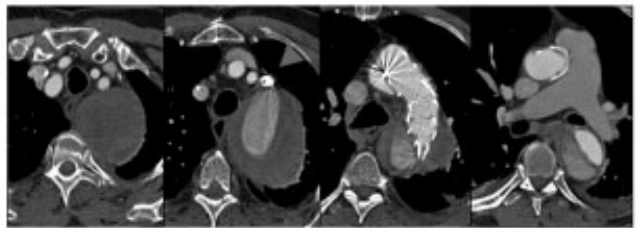

C
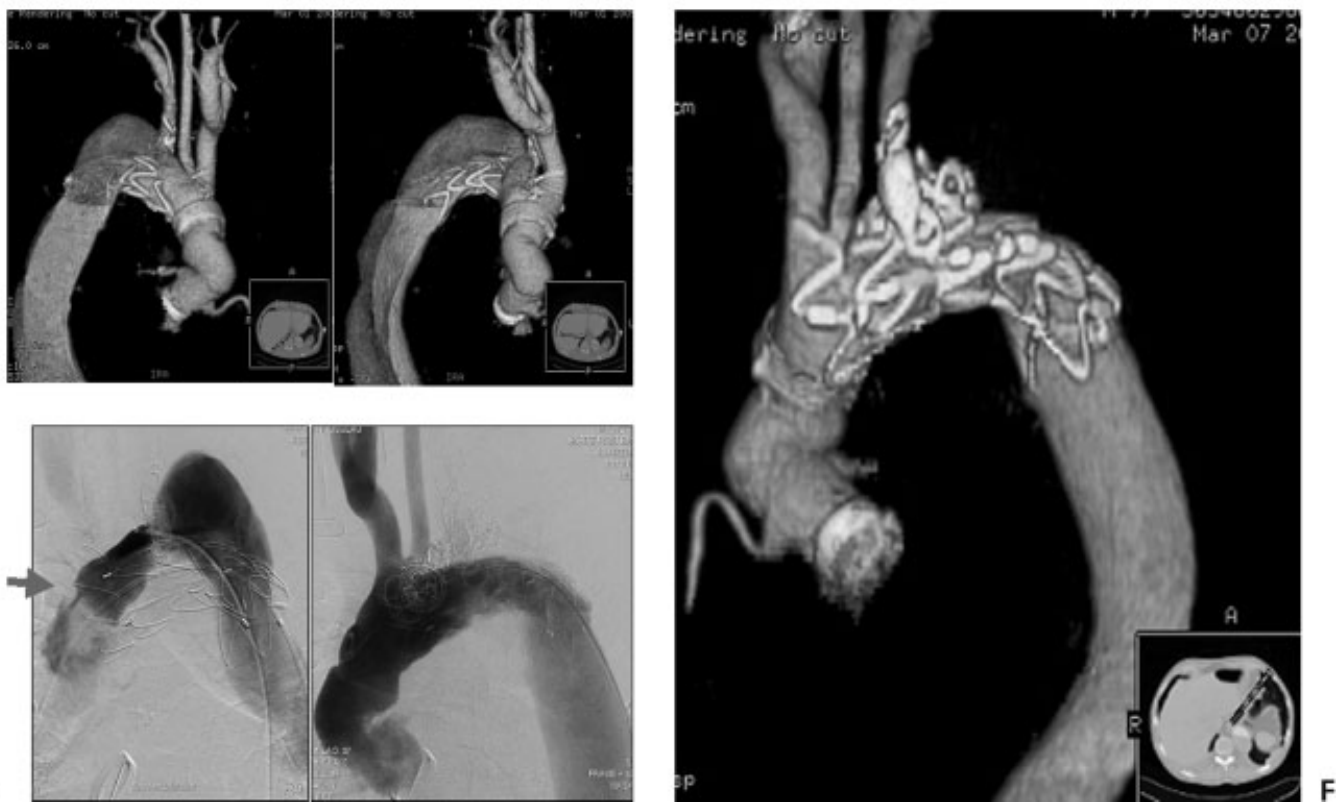

D

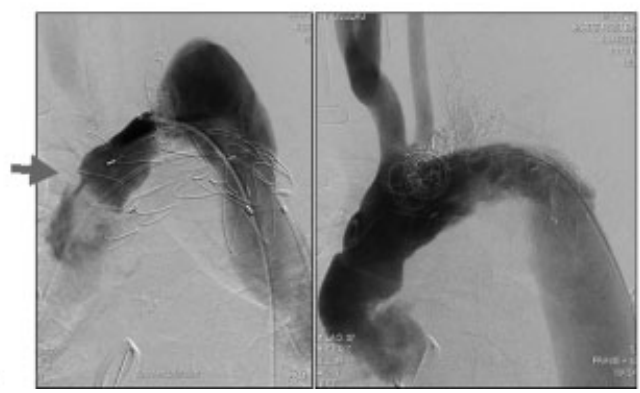

Figure 5 (A) Type A dissection in a 66-year-old man with previous aortic surgery. The false lumen is patent because of a proximal entry tear at the level of the arch. (B) A stent graft was placed, and during the same procedure the left subclavian artery (LSA) was coiled to prevent retrograde refilling with subsequent type II endoleak. (C) 3D postoperative computed tomography (CT) reconstructions show correct placement of the thoracic stent graft in the arch and coils positioned just at the origin of LSA, which looks distally patent. Persistent proximal flow was observed in the false lumen. (D) Selective angiography of the false lumen demonstrated a proximal entry tear at the level of the distal ascending aorta. (E) Extensive coiling of false lumen, with resultant complete thrombosis of false lumen at final angiographic control and postoperative CT scan. (F) Thrombosis of the false lumen is the final target of stent graft repair in an aortic dissection. To achieve it, it is sometimes necessary to perform embolization of collaterals or even the false lumen.

multicenter, randomized, controlled clinical study, was started in 2002, to compare the 1-year outcome of type B aortic dissection treated by stent-graft placement versus conventional antihypertensive therapy. ${ }^{65}$ Early results will be published in 2007 .

The mortality of IMH with involvement of the ascending aorta has been considered to be similar to that of aortic type A dissection; thus early surgical graft repair is the standard treatment in these patients. Conversely, in asymptomatic patients or those beyond 48 hours from the onset of symptoms with IMH located in the arch or descending aorta, watchful waiting and aggressive antihypertensive treatment appears a safe strategy; however, frequent follow-up imaging evaluations for evidence of intimal erosion or disease progression is required, and a low threshold for intervention should be maintained if symptoms recur. Classically, surgery is reserved for patients who develop complications such as persistent pain, penetrating atherosclerotic ulcer, signs of impending rupture, progressive maximal aortic wall thickness and compression of the true lumen, enlarging aortic diameter, or compromise of a major arterial branch. This conservative approach to type B IMH is supported by studies that document similar survival statistics for medical treatment and surgical repair in the setting of descending thoracic IMH. ${ }^{66}$

However, conventional open repair requiring graft interposition is associated with high morbidity and mortality rates, especially in this complicated setting in patients who typically are not ideal operative candidates because of advanced age and/or coexisting medical diseases. Consequently, less invasive strategies that rely 
on the endovascular placement of stent grafts to cover some extent of the IMH have been recently investigated for complicated IMH with promising initial results. ${ }^{67}$

Application of stent grafts to cases of uncomplicated type B IMH without associated intimal disruption or ulcer has not yet been reported. Another problem is the timing of the stent-graft insertion. Just after the start of the symptoms, in most of the cases the location of the intimal lesion or ulcer is not seen and one needs to cover a long segment of the aorta with a risk of paraplegia and an excessive cost. By waiting, most of the hematoma will disappear with no need for invasive treatment, and if an ulcer is seen it could be treated by a relatively short stent graft.

Multicenter studies are needed to refine management approaches to IMH and establish treatment guidelines that will improve outcomes and benefit patients beyond what is achieved currently. Efforts to extend applications of endovascular stent-graft technology to aortic dissection variants have focused predominantly on PAU. ${ }^{68-70}$ Because PAU is usually focal and almost always in the descending aorta, it is an ideal anatomical target for endovascular stent-graft repair. To minimize the risk of paraplegia, a short device could be used to locally seal and stabilize the lesion. In a meta-analysis of 54 patients accumulated from 13 studies, complete sealing of the ulcer was achieved in 94\%, neurological complications occurred in 6\%, and in-hospital mortality was $5 \%{ }^{71}$

\section{Ischemic Complications}

The extreme complexity of aortic dissections with associated ischemic complications is a tremendous therapeutic challenge. The decision about treatment is based on the pathophysiology of the lesions. The Stanford University group developed a phantom model for evaluation of the relative hemodynamic benefits of various interventions in aortic dissection. ${ }^{72}$ In this model, true lumen collapse largely depended on the ratios of inflow capacity to outflow capacity in the true and false aortic lumina. True lumen collapse was exacerbated by increasing the size of the primary aortic tear, occluding false lumen outflow branches, and increasing true lumen outflow by lowering the peripheral resistance of its outflow vessels. In the model, the best method of treating true lumen collapse was to diminish false lumen inflow by repairing the primary tear with a stent graft. A less effective but important alternative was to create communications between the true and false lumen distal to the level of the compromised branches. The investigators recommended the preferential use of the false lumen as the inflow source for branch vessel stents when possible to avoid increasing true lumen outflow (which might promote further true lumen collapse). One of the drawbacks of this method is the risk of a secondary occlusion of the stent if surgery or stent graft implantation is done later on the proximal entry tear, with a secondary thrombosis of the false lumen feeding the visceral branches.

In dynamic dissections, distal ischemia is caused by obstruction of one of the two channels responsible for distal outflow. This can be the true or false lumen and can occur without extension of the dissection into the affected aortic side branch. Endovascular treatment attempts to restore adequate perfusion through one of the two channels to the outflow tract. Implantation of a TSG in the true lumen at the level of the thoracic entry excludes the false lumen with subsequent decreased pressure and compression on the true lumen. Finally, blood flow through the true lumen is restored with adequate perfusion of aortic side branches. If exclusion of the false lumen does not reestablish sufficient flow at the level of the infrarenal aorta and/or the iliac arteries and if peripheral ischemia persists, fenestration or infrarenal stent placement could be used to rebalance arterial pressure in both channels. In the study by Dake et al comprising 28 ischemic complications, $100 \%$ of dynamic obstructions and $40 \%$ of combined static and dynamic obstructions could be corrected by means of TSG implantation. In the other cases ischemia was treated with stent placement and fixation of an intimal flap in the affected artery or by the fenestration technique. ${ }^{38}$ In other series the success rate of these revascularizations varies between 92 and 100\%. ${ }^{37,46}$

In static dissections, ischemia is caused by extension of the dissection until the level of the affected artery. If no reentry occurs distal to the affected artery, the true lumen is usually compressed by the false channel. Placement of a stent in the true lumen allows reopening and might reestablish flow in the true lumen. Lower limb ischemia caused by extension of the dissection in the abdominal aorta and iliac arteries can be treated either by an infrarenal stent or by the previously described technique of fenestration if thrombus is not present. The use of endovascular methods to treat complicated aortic dissection was first reported in 1990, but only two centers have reported a significant experience with these procedures. The University of Michigan's experience, in which 24 patients with aortic dissection (13, type A; 8, type B; 3, atypical) were treated with percutaneous balloon fenestration and/or stent placement, a total of $92 \%$ of the compromised vascular beds were successfully reperfused, with a $25 \% 30$-day mortality rate. ${ }^{46}$ At least three of the six deaths in this study were related to irreversible ischemia of abdominal organs. In addition, 2 of the 18 surviving patients (11\%) died from complications of false lumen enlargement during the follow-up period. In a later follow-up study, the same group reported decreased mortality rates after incorporating percutaneous revascularization procedures into a "surgical delay" strategy for selected patients with type A dissection and late-stage ischemic manifestations. The 
Stanford University group published their experience of 40 patients presenting with acute aortic dissection (10, type A; 30, type B) treated with fenestration and/or stent placement; $93 \%$ of patients were successfully with a $25 \%$ 30 -day mortality rate. ${ }^{37}$ Of the early survivors, 5 of 30 patients (17\%) died within the follow-up period, among which at least 1 patient had false lumen rupture.

Irreversible bowel ischemia at the time of intervention, type $\mathrm{A}$ aortic dissection, and patients with chronic aortic dissection seem to be independent predictors of 30 -day mortality. ${ }^{37,46,73}$ For all these reasons, and because the long-term patency of endovascular reconstruction in aortic dissection is not yet known, and also because these procedures do not address the possibility of future false lumen aneurysm formation, regular imaging follow-up is imperative.

\section{Conclusion}

Many new aspects about risk factors, clinical characteristics, diagnosis, and management of acute aortic dissection have been learned over the last decade. Furthermore, diverse percutaneous strategies to treat aortic syndromes are continuing to improve and evolve. Despite the relatively small number of patients reported in the literature and limited follow-up, endovascular techniques appear to be successful in the treatment of complications related to aortic dissections. Implantation of TSGs, with or without other endovascular procedures, is a potential and promising alternative to surgery in patients with complicated type B dissections in whom the vascular anatomy allows implantation of an endoprothesis. Those techniques might even be justified in cases of persistent ischemia after surgical intervention. The techniques described also represent an alternative therapy for nonsurgical candidates. Their application on a larger scale cannot be considered before further studies compare the long-term results of the endovascular approach with the outcome of medical treatment for uncomplicated type B dissections.

\section{REFERENCES}

1. Meszaros I, Morocz J, Szlavi J, et al. Epidemiology and clinicopathology of aortic dissection. Chest 2000;117:12711278

2. Bickerstaff LK, Pairolero PC, Hollier LH, et al. Thoracic aortic aneurysms: a population-based study. Surgery 1982;92: 1103-1108

3. Clouse WD, Hallett JW Jr, Schaff HV, et al. Acute aortic dissection: population-based incidence compared with degenerative aortic aneurysm rupture. Mayo Clin Proc 2004;79: 176-180

4. Hagan PG, Nienaber CA, Isselbacher EM, et al. The International Registry of Acute Aortic Dissection (IRAD): new insights into an old disease. JAMA 2000;283:897-903
5. Nienaber CA, Fattori R, Mehta RH, et al. Gender-related differences in acute aortic dissection. Circulation 2004;109: 3014-3021

6. Nienaber CA, Sievers HH. Intramural hematoma in acute aortic syndrome: more than one variant of dissection? Circulation 2002;106:284-285

7. Moizumi Y, Komatsu T, Motoyoshi N, Tabayashi K. Clinical features and long-term outcome of type A and type $\mathrm{B}$ intramural hematoma of the aorta. J Thorac Cardiovasc Surg 2004;127:421-427

8. Movsowitz HD, Lampert C, Jacobs LE, Kotler MN. Penetrating atherosclerotic aortic ulcers. Am Heart J 1994; 128:1210-1217

9. Ganaha F, Miller DC, Sugimoto K, et al. Prognosis of aortic intramural hematoma with and without penetrating atherosclerotic ulcer: a clinical and radiological analysis. Circulation 2002;106:342-348

10. Lesauskaite V, Tanganelli P, Sassi C, et al. Smooth muscle cells of the media in the dilatative pathology of ascending thoracic aorta: morphology, immunoreactivity for osteopontin, matrix metalloproteinases, and their inhibitors. Hum Pathol 2001;32:1003-1011

11. DeBakey ME, Beall AC Jr, Cooley DA, et al. Dissecting aneurysms of the aorta. Surg Clin North Am 1966;46:10451055

12. Daily PO, Trueblood HW, Stinson EB, Wuerflein RD, Shumway NE. Management of acute aortic dissections. Ann Thorac Surg 1970;10:237-247

13. Erbel R, Oelert H, Meyer J, et al. Effect of medical and surgical therapy on aortic dissection evaluated by transesophageal echocardiography: implications for prognosis and therapy. The European Cooperative Study Group on Echocardiography. Circulation 1993;87:1604-1615

14. Miller DC, Mitchell RS, Oyer PE, Stinson EB, Jamieson SW, Shumway NE. Independent determinants of operative mortality for patients with aortic dissections. Circulation 1984;70:I153-I164

15. Erbel R, Alfonso F, Boileau C, et al. Diagnosis and management of aortic dissection. Eur Heart J 2001;22: 1642-1681

16. Mehta RH, Suzuki T, Hagan PG, et al. Predicting death in patients with acute type a aortic dissection. Circulation 2002; 105:200-206

17. DeBakey ME, McCollum $\mathrm{CH}$, Crawford ES, et al. Dissection and dissecting aneurysms of the aorta: twentyyear follow-up of five hundred twenty-seven patients treated surgically. Surgery 1982;92:1118-1134

18. Glower DD, Fann JI, Speier RH, et al. Comparison of medical and surgical therapy for uncomplicated descending aortic dissection. Circulation 1990;82(suppl):IV39-IV46

19. Schor JS, Yerlioglu ME, Galla JD, Lansman SL, Ergin MA, Griepp RB. Selective management of acute type B aortic dissection: long-term follow-up. Ann Thorac Surg 1996;61: 1339-1341

20. Svensson LG, Crawford ES, Hess KR, Coselli JS, Safi HJ. Dissection of the aorta and dissecting aortic aneurysms: improving early and long-term surgical results. Circulation 1990;82(suppl):IV24-IV38

21. Fann JI, Miller DC. Aortic dissection. Ann Vasc Surg 1995; 9:311-323

22. Suzuki T, Mehta RH, Ince $H$, et al. Clinical profiles and outcomes of acute type B aortic dissection in the current era: lessons from the International Registry of Aortic 
Dissection (IRAD). Circulation 2003;108(suppl 1):II312II317

23. Fann JI, Harris GE, Mitchell RS, et al. Treatment of patients with aortic dissection presenting with peripheral vascular complications. Ann Surg 1990;212:705-713

24. Cambria RP, Brewster DC, Gertler J, et al. Vascular complications associated with spontaneous aortic dissection. J Vasc Surg 1988;7:199-209

25. Laas J, Heinemann M, Schaefers HJ, Daniel W, Borst HG. Management of thoracoabdominal malperfusion in aortic dissection. Circulation 1991;84(suppl):III20-III24

26. Bossone E, Rampoldi V, Nienaber CA, et al. Usefulness of pulse deficit to predict in-hospital complications and mortality in patients with acute type A aortic dissection. Am J Cardiol 2002;89:851-855

27. Walker PJ, Dake MD, Mitchell RS, Miller DC. The use of endovascular techniques for the treatment of complications of aortic dissection. J Vasc Surg 1993;18:1042-1051

28. Elefteriades JA, Hartleroad J, Gusberg RJ, et al. Long-term experience with descending aortic dissection: the complication-specific approach. Ann Thorac Surg 1992;53:11-20; discussion 20-21

29. Wheat MW Jr. Acute dissection of the aorta. Cardiovasc Clin 1987;17:241-262

30. Richter GM, Allenberg JR, Schumacher H, Hansmann J, Vahl C, Hagl S. [Aortic dissection-when operative treatment, when endoluminal therapy?]. Radiologe 2001;41:660667

31. Doroghazi RM, Slater EE, DeSanctis RW, Buckley MJ, Austen WG, Rosenthal S. Long-term survival of patients with treated aortic dissection. J Am Coll Cardiol 1984;3: 1026-1034

32. Sueyoshi E, Sakamoto I, Hayashi K, Yamaguchi T, Imada T. Growth rate of aortic diameter in patients with type B aortic dissection during the chronic phase. Circulation 2004; 110(suppl 1):II256-II261

33. Sawhney NS, DeMaria AN, Blanchard DG. Aortic intramural hematoma: an increasingly recognized and potentially fatal entity. Chest 2001;120:1340-1346

34. Nienaber CA, Richartz BM, Rehders T, Ince H, Petzsch M. Aortic intramural haematoma: natural history and predictive factors for complications. Heart 2004;90:372-374

35. Coady MA, Rizzo JA, Hammond GL, Pierce JG, Kopf GS, Elefteriades JA. Penetrating ulcer of the thoracic aorta: what is it? How do we recognize it? How do we manage it? J Vasc Surg 1998;27:1006-1015; discussion 1015-1016

36. Tittle SL, Lynch RJ, Cole PE, et al. Midterm follow-up of penetrating ulcer and intramural hematoma of the aorta. J Thorac Cardiovasc Surg 2002;123:1051-1059

37. Slonim SM, Miller DC, Mitchell RS, Semba CP, Razavi MK, Dake MD. Percutaneous balloon fenestration and stenting for life-threatening ischemic complications in patients with acute aortic dissection. J Thorac Cardiovasc Surg 1999;117:1118-1126

38. Dake MD, Kato N, Mitchell RS, et al. Endovascular stentgraft placement for the treatment of acute aortic dissection. N Engl J Med 1999;340:1546-1552

39. Nienaber CA, Fattori R, Lund G, et al. Nonsurgical reconstruction of thoracic aortic dissection by stent-graft placement. N Engl J Med 1999;340:1539-1545

40. Beregi JP, Prat A, Gaxotte V, Delomez M, McFadden EP. Endovascular treatment for dissection of the descending aorta. Lancet 2000;356:482-483
41. Kato M, Matsuda T, Kaneko M, et al. Outcomes of stentgraft treatment of false lumen in aortic dissection. Circulation 1998;98(suppl):II305-II311; discussion II311-312

42. Williams DM, Andrews JC, Marx MV, Abrams GD. Creation of reentry tears in aortic dissection by means of percutaneous balloon fenestration: gross anatomic and histologic considerations. J Vasc Interv Radiol 1993;4:75-83

43. Bernard Y, Zimmermann H, Chocron S, et al. False lumen patency as a predictor of late outcome in aortic dissection. Am J Cardiol 2001;87:1378-1382

44. Hausegger KA, Oberwalder $\mathrm{P}$, Tiesenhausen $\mathrm{K}$, et al. Intentional left subclavian artery occlusion by thoracic aortic stent-grafts without surgical transposition. J Endovasc Ther 2001;8:472-476

45. Rehders TC, Petzsch M, Ince H, et al. Intentional occlusion of the left subclavian artery during stent-graft implantation in the thoracic aorta: risk and relevance. J Endovasc Ther 2004; 11:659-666

46. Williams DM, Lee DY, Hamilton BH, et al. The dissected aorta: percutaneous treatment of ischemic complications: principles and results. J Vasc Interv Radiol 1997;8:605-625

47. Slonim SM, Nyman U, Semba CP, Miller DC, Mitchell RS, Dake MD. Aortic dissection: percutaneous management of ischemic complications with endovascular stents and balloon fenestration. J Vasc Surg 1996;23:241-251; discussion 251253

48. Chavan A, Hausmann D, Dresler C, et al. Intravascular ultrasound-guided percutaneous fenestration of the intimal flap in the dissected aorta. Circulation 1997;96:2124-2127

49. Yunoki K, Uchida H, Sano S, Shimizu N. Effects of endoluminal stent-grafts on acute aortic dissection in dogs. Acta Med Okayama 1998;52:89-95

50. Lopera J, Patino JH, Urbina C, et al. Endovascular treatment of complicated type-B aortic dissection with stent-grafts: midterm results. J Vasc Interv Radiol 2003;14:195-203

51. Palma JH, de Souza JA, Rodrigues Alves CM, Carvalho AC, Buffolo E. Self-expandable aortic stent-grafts for treatment of descending aortic dissections. Ann Thorac Surg 2002; 73:1138-1141; discussion 1141-1142

52. Taylor PR, Bell RE, Reidy JF. Endovascular treatment of acute type B aortic dissection. Acta Chir Belg 2004;104:513518

53. Fann JI, Miller DC. Endovascular treatment of descending thoracic aortic aneurysms and dissections. Surg Clin North Am 1999;79:551-574

54. Kato N, Hirano T, Shimono T, et al. Treatment of chronic type $\mathrm{B}$ aortic dissection with endovascular stent-graft placement. Cardiovasc Intervent Radiol 2000;23:60-62

55. Kato N, Shimono T, Hirano T, et al. Midterm results of stent-graft repair of acute and chronic aortic dissection with descending tear: the complication-specific approach. J Thorac Cardiovasc Surg 2002;124:306-312

56. Shimono T, Kato N, Yasuda F, et al. Transluminal stentgraft placements for the treatments of acute onset and chronic aortic dissections. Circulation 2002;106(suppl 1): I241-I247

57. Svensson LG, Hess KR, Coselli JS, Safi HJ. Influence of segmental arteries, extent, and atriofemoral bypass on postoperative paraplegia after thoracoabdominal aortic operations. J Vasc Surg 1994;20:255-262

58. Eggebrecht H, Nienaber CA, Neuhauser M, et al. Endovascular stent-graft placement in aortic dissection: a metaanalysis. Eur Heart J 2006;27:489-498 
59. Umana JP, Miller DC, Mitchell RS. What is the best treatment for patients with acute type B aortic dissections: medical, surgical, or endovascular stent-grafting? Ann Thorac Surg 2002;74:S1840-S1843; discussion S1857-S1863

60. Gaxotte V, Thony F, Rousseau H, et al. Midterm results of aortic diameter outcomes after thoracic stent-graft implantation for aortic dissection: a multicenter study. J Endovasc Ther 2006;13:127-138

61. Akutsu K, Nejima J, Kiuchi K, et al. Effects of the patent false lumen on the long-term outcome of type B acute aortic dissection. Eur J Cardiothorac Surg 2004;26:359-366

62. Nienaber CA, Kische S, Zeller T, et al. Provisional extension to induce complete attachment after stent-graft placement in type B aortic dissection: the PETTICOAT concept. J Endovasc Ther 2006;13:738-746

63. Shimono T, Kato N, Tokui T, et al. Endovascular stent-graft repair for acute type $\mathrm{A}$ aortic dissection with an intimal tear in the descending aorta. J Thorac Cardiovasc Surg 1998; 116:171-173

64. Kato N, Shimono T, Hirano T, Ishida M, Yada I, Takeda K. Transluminal placement of endovascular stent-grafts for the treatment of type A aortic dissection with an entry tear in the descending thoracic aorta. J Vasc Surg 2001;34:1023-1028

65. Nienaber CA, Zannetti S, Barbieri B, et al. INvestigation of STEnt grafts in patients with type B Aortic Dissection: design of the INSTEAD trial-a prospective, multicenter, European randomized trial. Am Heart J 2005;149:592-599

66. Harris KM, Braverman AC, Gutierrez FR, Barzilai B, Davila-Roman VG. Transesophageal echocardiographic and clinical features of aortic intramural hematoma. J Thorac Cardiovasc Surg 1997;114:619-626

67. Schoder M, Grabenwoger M, Holzenbein T, et al. Endovascular stent-graft repair of complicated penetrating atherosclerotic ulcers of the descending thoracic aorta. J Vasc Surg 2002;36:720-726

68. Kos X, Bouchard L, Otal P, et al. Stent-graft treatment of penetrating thoracic aortic ulcers. J Endovasc Ther 2002; 9(suppl 2):II25-II31

69. Brittenden J, McBride K, McInnes G, Gillespie IN, Bradbury AW. The use of endovascular stents in the treatment of penetrating ulcers of the thoracic aorta. J Vasc Surg 1999;30: 946-949

70. Demers P, Miller DC, Mitchell RS, Kee ST, Chagonjian L, Dake MD. Stent-graft repair of penetrating atherosclerotic ulcers in the descending thoracic aorta: mid-term results. Ann Thorac Surg 2004;77:81-86

71. Eggebrecht H, Baumgart D, Schmermund A, et al. Penetrating atherosclerotic ulcer of the aorta: treatment by endovascular stent-graft placement. Curr Opin Cardiol 2003; 18:431-435

72. Chung JW, Elkins C, Sakai T, et al. True-lumen collapse in aortic dissection: part II. Evaluation of treatment methods in phantoms with pulsatile flow. Radiology 2000;214: 99-106

73. Vedantham S, Picus D, Sanchez LA, et al. Percutaneous management of ischemic complications in patients with type-B aortic dissection. J Vasc Interv Radiol 2003;14:181194 